\title{
CARTOX Grade 0
}

National Cancer Institute

\section{Source}

National Cancer Institute. CART OX Grade O. NCI Thesaurus. Code C156291.

A score of 10 on the CART OX-10 assessment, indicating no neurotoxicity. 